\title{
Proximate order and lower type of entire gap power series
}

\author{
By H. S. KasAna
}

(Received May 31, 2001)

(from Nagoya Mathematical Journal)

\begin{abstract}
The paper deals with the characterization of lower $(p, q)$-type with respect to a proximate order of an entire function in terms of coefficients and exponents of its power series expansion. In particular, the problem proposed by Bajpai et al. [Trans. Amer. Math. Soc. (1975)], and subsequently, left open by Juneja et al. also [J. Reine Angew. Math. (1977)] has been solved.
\end{abstract}

\section{Introduction}

Let $f(z)=\sum_{n=0}^{\infty} a_{n} z^{\lambda_{n}}$ be a nonconstant entire function, where $\lambda_{o}=0$ and $\left\{\lambda_{n}\right\}_{n=1}^{\infty}$ is a strictly increasing sequence of positive integers such that $a_{n} \neq 0$ for $n=1,2, \ldots$. We set $M(r) \equiv M(r, f)=\max _{|z|=r}|f(z)| ; \mu(r) \equiv \mu(r, f)=$ $\max _{n \geq 1}\left\{\left|a_{n}\right| r^{\lambda_{n}}\right\}$ and $\nu(r) \equiv \nu(r, f)=\max \left\{\lambda_{n}: \mu(r)=\left|a_{n}\right| r^{\lambda_{n}}\right\}$. Then $M(r)$, $\mu(r)$ and $\nu(r)$ are called the maximum modulus, maximum term and rank of the maximum term, respectively of $f(z)$ for $|z|=r$. The concepts of index-pair $(p, q)$, $p \geq q \geq 1,(p, q)$-orders and $(p, q)$-types were introduced by Juneja et al. ([2], [3]). The growth of an entire function $f(z)$ can be studied in terms of its $(p, q)$-orders and $(p, q)$-types. However, these concepts are inadequate for comparing the growth of those entire functions which are of the same $(p, q)$-orders but of infinite $(p, q)$ types. For a refinement of the above scale we utilize the concept of proximate order defined as follows:

Definition 1. A positive function $\rho(r)$ defined on $\left[r_{o}, \infty\right), r_{o}>\exp ^{[q-1]} 1$ is said to be a proximate order of an entire function with index-pair $(p, q)$ if the following conditions are satisfied, where $\exp ^{[0]} x=x$ and $\exp ^{[q]} x=\exp \left(\exp ^{[q-1]}\right) x$.

(i) $\rho(r) \rightarrow \rho(p, q) \equiv \rho$ as $r \rightarrow \infty, b<\rho<\infty$;

(ii) $\Lambda_{[q]}(r) \rho^{\prime}(r) \rightarrow 0$ as $r \rightarrow \infty$, where $\rho^{\prime}(r)$ denotes the derivative of $\rho(r)$, $b=1$ if $p=q, b=0$ if $p>q$ and, for convenience, $\Lambda_{[q]}(r)=\prod_{i=0}^{q} \log ^{[i]} r$, and $\log ^{[i]} x$ is defined by $\log ^{[0]} x=x$ and $\log ^{[q]} x=\log \left(\log { }^{[q-1]}\right) x$.

1991 Mathematics Subject Classification. Primary 30D15; Secondary 30B20.

Key words and Phrases. Index-pair, proximate order and generalized $(p, q)$-types. 
Definition 2. With index-pair $(p, q), p \geq q \geq 1$, the generalized $(p, q)$-type $T^{*}$ and the generalized lower $(p, q)$-type $t^{*}$ of $f(z)$ in reference to a proximate order $\rho(r)$ (satisfying (i) and (ii)) are defined as

$$
\lim _{r \rightarrow \infty} \sup \frac{\log ^{[p-1]} M(r)}{\left(\log ^{[q-1]} r\right)^{\rho(r)}}=\begin{aligned}
& T^{*}(p, q) \equiv T^{*} \\
& t^{*}(p, q) \equiv t^{*}
\end{aligned} ; \quad 0 \leq t^{*} \leq T^{*} \leq \infty .
$$

Clearly, the proximate order and generalized lower $(p, q)$-type of an entire function are not uniquely determined. The existence theorem for such comparison functions on $(p, q)$-scale has been established in [4] and [5].

Definition 3. An entire function $f(z)$ is said to be of perfectly regular $(p, q)$ growth in reference to its proximate order if $0<t^{*}=T^{*}<\infty$.

Juneja et al. [3] proved the following:

TheOREm A. Let $f(z)$ be an entire function having $(p, q)$-order $\rho(b<\rho<$ $\infty)$ and lower $(p, q)$-type $t$ such that

(i) $\psi(n)=\left|a_{n} / a_{n+1}\right|^{1 /\left(\lambda_{n+1}-\lambda_{n}\right)}$ forms a nondecreasing function of $n$ for $n>n_{o}$,

(ii) $\log ^{[p-2]} \lambda_{n-1} \simeq \log ^{[p-2]} \lambda_{n}$ as $n \rightarrow \infty$.

Then

$$
\frac{t}{M}=\liminf _{n \rightarrow \infty} \frac{\log ^{[p-2]} \lambda_{n-1}}{\left(\log ^{[q-1]}\left|a_{n}\right|^{-1 / \lambda_{n}}\right)^{\rho-A}},
$$

where $A=1$ if $q=2, A=0$ if $q \neq 2$, and

$$
M \equiv M(p, q)= \begin{cases}(\rho-1)^{\rho-1} / \rho^{\rho} & \text { if }(p, q)=(2,2) \\ 1 / e \rho & \text { if }(p, q)=(2,1) \\ 1 & \text { otherwise }\end{cases}
$$

This theorem was proved by Bajpai et al. [1] basically for the entire functions of Sato growth [9], i.e., $(p, q)=(p, 1)$ and they raised a question whether the asymptotic condition (ii) on exponents in Theorem A can be weakened. In a subsequent paper, Juneja et al. [3] extended the theorem for index-pair $(p, q)$ and again left this question open. In this paper we characterize lower $(p, q)$-type with respect to a proximate order in terms of coefficients and exponents of power series (with gaps) which proves Theorem A under weaker conditions. 


\section{Auxiliary results}

This section consists of some auxiliary results which will be utilized in the sequel.

Since $\left(\log { }^{[q-1]} r\right)^{\rho(r)-A}$ is a monotonically increasing function [6] of $r$ for $r>$ $r_{o}$, we can define $\phi(x)$ to be the unique solution of the equation

$$
x=\left(\log ^{[q-1]} r\right)^{\rho(r)-A} \Longleftrightarrow \phi(x)=\log ^{[q-1]} r,
$$

where $A=1$, if $(p, q)=(2,2)$ and $A=0$ otherwise.

LEMma 1. For the function $\phi(x)$ in (1.1), we have

$$
\lim _{x \rightarrow \infty} \frac{\phi(\eta x)}{\phi(x)}=\eta^{1 /(\rho-A)}, \quad \text { uniformly for } 0<\eta<\infty
$$

Proof. The proof of this lemma is available in [8].

Lemma 2. Let $f(z)$ be an entire function of $(p, q)$-order $\rho(b<\rho<\infty)$ and generalized lower $(p, q)$-type $t^{*}$, in reference to a proximate order $\rho(r)$. Then

$$
\liminf _{r \rightarrow \infty} \frac{\log ^{[p-1]} \mu(r)}{\left(\log ^{[q-1]} r\right)^{\rho(r)}}=t^{*}
$$

Proof. This lemma is an outcome of Definition 2 as well as the following inequality which is due to Lockhart and Straus [7],

$$
\mu(r)<M(r)<\left(\frac{4 r+\varepsilon}{\varepsilon}\right)\left(1+\log \frac{\mu(r+\varepsilon)}{\mu(r)}\right) \mu(r) .
$$

Lemma 3. Let $f(z)$ be an entire function of $(p, q)$-order $\rho(b<\rho<\infty)$ and generalized lower $(p, q)$-type $t^{*}$, in reference to a proximate order $\rho(r)$. Then, for $p \geq 3$,

$$
\liminf _{r \rightarrow \infty} \frac{\log ^{[p-2]} \nu(r)}{\left(\log ^{[q-1]} r\right)^{\rho(r)}}=t^{*}
$$

Proof. It is well known that $\log \mu(r)$ is an increasing convex function of $\log r$ for $r>r_{o}$ and has the representation

$$
\log \mu(r)=\log \mu\left(r_{o}\right)+\int_{r_{o}}^{r} \frac{\nu(x)}{x} d x
$$


As $\nu(r)$ is an increasing function of $r$, we observe that

$$
\log \mu(r) \leq O(1)+\nu(r) \log r,
$$

and by the property, $\log ^{[p]}(x+y) \leq \log ^{[p]} x+\log ^{[p]} y$, for $x, y>\exp ^{[p]} 1$,

$$
\log ^{[p-1]} \mu(r) \leq \log ^{[p-2]} \nu(r)+\log ^{[p-1]} r, \quad \text { for } p \geq 3 .
$$

Taking limits and considering Lemma 2, we have

$$
t^{*} \leq \liminf _{r \rightarrow \infty} \frac{\log ^{[p-2]} \nu(r)}{\left(\log { }^{[q-1]} r\right)^{\rho(r)}} .
$$

Also, (1.3) implies $\log \mu(e r) \geq \nu(r)$, which gives the reverse inequality to (1.4) and hence the lemma.

\section{Main results}

In this section we state and prove our results. To avoid repetitions, we shall assume throughout that $f(z)=\sum_{n=0}^{\infty} a_{n} z^{\lambda_{n}}$ is an entire function with index-pair $(p, q),(p, q)$-order $\rho(b<\rho<\infty)$ and generalized lower $(p, q)$-type $t^{*}$ with respect to a proximate order $\rho(r)$ satisfying (i) and (ii). We prove

Proposition 1. For an entire function $f(z)$ and any increasing sequence $\left\{n_{k}\right\}$ of positive integers such that $n_{k} \rightarrow \infty$ as $k \rightarrow \infty$, we have

$$
\frac{t^{*}}{M} \geq G \liminf _{k \rightarrow \infty}\left[\frac{\phi\left(\log ^{[p-2]} \lambda_{n_{k-1}}\right)}{\log { }^{[q-1]}\left|a_{n_{k}}\right|^{-1 / \lambda_{n_{k}}}}\right]^{\rho-A},
$$

where $t^{*}$ is the generalized lower $(p, q)$-type with respect to a proximate order $\rho(r)$, $A$ and $M$ have the same meaning as in Theorem $\mathrm{A}$ and

$$
G \equiv G\left(\left\{n_{k}\right\}\right)= \begin{cases}\liminf _{k \rightarrow \infty}\left(\lambda_{n_{k-1}} / \lambda_{n_{k}}\right)^{1 /(\rho-1)} & \text { if }(p, q)=(2,2) \\ 1 & \text { if }(p, q) \neq(2,2) .\end{cases}
$$

Proof. Let us put

$$
\liminf _{k \rightarrow \infty}\left[\frac{\phi\left(\log ^{[p-2]} \lambda_{n_{k-1}}\right)}{\log ^{[q-1]}\left|a_{n_{k}}\right|^{-1 / \lambda_{n_{k}}}}\right]^{\rho-A}=B
$$


Obviously, $B \geq 0$. If $B=0$ the proof of (2.1) is trivial. Hence, let $B>0$. Then, for any arbitrary $\varepsilon, 0<\varepsilon<B$ and $\forall k>k_{o}(\varepsilon)$, we have

$$
\frac{\phi\left(\log ^{[p-2]} \lambda_{n_{k-1}}\right)}{\log ^{[q-1]}\left|a_{n_{k}}\right|^{-1 / \lambda_{n_{k}}}}>(B-\varepsilon)^{1 /(\rho-A)}
$$

or

$$
\log \left|a_{n_{k}}\right|>-\lambda_{n_{k}} \exp ^{[q-2]}\left(\frac{\phi\left(\log ^{[p-2]} \lambda_{n_{k-1}}\right)}{(B-\varepsilon)^{1 /(\rho-A)}}\right) .
$$

Keeping in view $k>k_{o}(\varepsilon)$ and $r>0$, we use Cauchy's inequality in above and derive

$$
\log M(r)>\lambda_{n_{k}} \log r-\lambda_{n_{k}} \exp ^{[q-2]}\left(\frac{\phi\left(\log ^{[p-2]} \lambda_{n_{k-1}}\right)}{(B-\varepsilon)^{1 /(\rho-A)}}\right) .
$$

Now, for $(p, q)=(2,1)$, we choose a sequence $\left\{r_{k}\right\}$ satisfying

$$
r_{k}=\frac{\phi\left(e \lambda_{n_{k-1}}\right)}{(B-\varepsilon)^{1 / \rho}}, \quad k>k_{o}
$$

When $r_{k} \leq r<r_{k+1},(2.2)$ leads to

$$
\log M(r)>\lambda_{n_{k}} \log r_{k}-\lambda_{n_{k}} \log \left(\frac{\phi\left(\lambda_{n_{k-1}}\right)}{(B-\varepsilon)^{1 / \rho}}\right) \simeq \frac{\lambda_{n_{k}}}{\rho},
$$

because $\phi\left(e \lambda_{n_{k-1}}\right) \simeq \phi\left(\lambda_{n_{k-1}}\right) e^{1 / \rho}$ as $k \rightarrow \infty$ by (1.2). Using the value of $\lambda_{n_{k}}$ from (2.3) and definition of $\phi(x)$, we have

$$
\begin{aligned}
\log M(r) & >\left(\frac{B-\varepsilon}{e}\right)^{\rho(r) / \rho} \frac{r_{k+1}^{\rho(r)}}{\rho} \\
& >(B-\varepsilon)^{\rho(r) / \rho} \frac{r^{\rho(r)}}{e \rho} \text { for } r_{k} \leq r<r_{k+1} .
\end{aligned}
$$

Dividing by $r^{\rho(r)}$ and proceeding to limits as $r \rightarrow \infty$, we get

$$
\liminf _{r \rightarrow \infty} \frac{\log M(r)}{r^{\rho(r)}}=t^{*} \geq \frac{B}{e \rho} .
$$

For the case $(p, q)=(2,2)$, we choose the sequence $\left\{r_{k}\right\}$ satisfying the relation

$$
\log r_{k}=\frac{\rho}{\rho-1} \cdot \frac{\phi\left(\lambda_{n_{k-1}}\right)}{(B-\varepsilon)^{1 /(\rho-1)}} .
$$


When $r_{k} \leq r<r_{k+1}$, we have from $(2.2)$

$$
\begin{aligned}
\log M(r) & >\lambda_{n_{k}} \log r_{k}-\lambda_{n_{k}} \frac{\phi\left(\lambda_{n_{k-1}}\right)}{(B-\varepsilon)^{1 /(\rho-1)}} \\
& =\frac{\lambda_{n_{k}} \phi\left(\lambda_{n_{k-1}}\right)}{(\rho-1)(B-\varepsilon)^{1 /(\rho-1)}} .
\end{aligned}
$$

From (2.5), we have

$$
\phi\left(\frac{\lambda_{n_{k}}}{B-\varepsilon}\right)=\log r_{k+1}^{(\rho-1) / \rho} .
$$

Taking (1.1) into consideration this yields

$$
\frac{\lambda_{n_{k}}}{B-\varepsilon}=\left(\log r_{k+1}^{(\rho-1) / \rho}\right)^{\rho\left(r_{k+1}^{(\rho-1) / \rho}\right)-1}
$$

The asymptotic property of $\rho(r)$ when $(p, q)=(2,2)$,

$$
\left(\log r^{k}\right)^{\rho\left(r^{k}\right)} \simeq k^{\rho}(\log r)^{\rho(r)} \text { as } r \rightarrow \infty,
$$

coupled with (2.7), yields

$$
\lambda_{n_{k}} \simeq(B-\varepsilon)^{\rho /(\rho-1)}\left(\frac{\rho-1}{\rho}\right)^{\rho} \frac{\left(\log r_{k+1}\right)^{\rho\left(r_{k+1}\right)}}{\phi\left(\lambda_{n_{k}}\right)} .
$$

Using the above estimate in (2.6), when $r_{k} \leq r<r_{k+1}$, we find that

$$
\log M(r)>(B-\varepsilon) \frac{(\rho-1)^{\rho-1}}{\rho^{\rho}} \frac{(\log r)^{\rho(r)} \phi\left(\lambda_{n_{k-1}}\right)}{\phi\left(\lambda_{n_{k}}\right)}
$$

or

$$
\frac{\log M(r)}{(\log r)^{\rho(r)}}>(B-\varepsilon) \frac{(\rho-1)^{\rho-1}}{\rho^{\rho}} \frac{\phi\left(\lambda_{n_{k-1}}\right)}{\phi\left(\lambda_{n_{k}}\right)} .
$$

Proceeding to limits as $k \rightarrow \infty$ and hence $r \rightarrow \infty$ (in view of definition of $G$ ),

$$
t^{*} \geq G \frac{B(\rho-1)^{\rho-1}}{\rho^{\rho}} .
$$

Let us now consider the case when $(p, q) \neq(2,1)$ and $(p, q) \neq(2,2)$. For this we define the sequence $\left\{r_{k}\right\}$ as

$$
\log r_{k}=1+\exp ^{[q-2]}\left(\frac{\phi\left(\log ^{[p-2]} \lambda_{n_{k-1}}\right)}{(B-\varepsilon)^{1 / \rho}}\right) .
$$


From (2.2), we get

$$
\log M(r)>\lambda_{n_{k}}
$$

or

$$
\log ^{[p-1]} M(r)>(B-\varepsilon)^{\rho(r) / \rho}\left(\log [q-1]\left(\frac{r_{k+1}}{e}\right)\right)^{\rho(r)}
$$

or

$$
\frac{\log ^{[p-1]} M(r)}{\left(\log ^{[q-1]}(r / e)\right)^{\rho(r)}}>(B-\varepsilon)^{\rho(r) / \rho} .
$$

Keeping in view, $\rho(r) \rightarrow \rho$ as $r \rightarrow \infty$ the above inequality gives $t^{*} \geq B$. Combining this with the inequalities (2.4) and (2.8), we get the required result.

Proposition 2. For an entire function $f(z)$, if $\psi(n)=\left|a_{n} / a_{n+1}\right|^{1 /\left(\lambda_{n+1}-\lambda_{n}\right)}$ forms a nondecreasing function of $n \forall n>n_{o}$, then

$$
t^{*} \leq \liminf _{n \rightarrow \infty}\left[\frac{\phi\left(\log ^{[p-2]} \lambda_{n-1}\right)}{\log ^{[q-1]}\left|a_{n}\right|^{-1 / \lambda_{n}}}\right]^{\rho}, \quad p \geq 3,
$$

and, for $p=2$, we have

$$
\frac{t^{*}}{M} \leq \liminf _{n \rightarrow \infty}\left[\frac{\phi\left(\lambda_{n}\right)}{\log ^{[A]}\left|a_{n}\right|^{-1 / \lambda_{n}}}\right]^{\rho-A},
$$

where $M$ is defined in Theorem A and $t^{*}$ is the generalized lower $(p, q)$-type as in Proposition 1 .

Proof. From Lemma 2, for given $\varepsilon>0$ and $r>r_{o}$, we have

$$
\log ^{[p-1]} \mu(r)>\left(t^{*}-\varepsilon\right)\left(\log ^{[q-1]} r\right)^{\rho(r)} .
$$

Since $\psi(n)$ is a nondecreasing function of $n$, the maximum term of $f(z)$ is

$$
\mu(r)=\left|a_{n}\right| r^{\lambda_{n}} \quad \text { when } \quad \psi(n-1) \leq r<\psi(n) .
$$

Hence (2.11) can be written as

$$
\log \left|a_{n}\right|>\exp ^{[p-2]}\left(\left(t^{*}-\varepsilon\right)(\log [q-1] r)^{\rho(r)}\right)-\lambda_{n} \log r .
$$

For $(p, q)=(2,1),(2.13)$ is reduced to

$$
\log \left|a_{n}\right|>\left(t^{*}-\varepsilon\right) r^{\rho(r)}-\lambda_{n} \log r .
$$


Now $r$ is chosen such that

$$
r^{\rho(r)}=\lambda_{n} / \rho\left(t^{*}-\varepsilon\right) \Longleftrightarrow r=\phi\left(\frac{\lambda_{n}}{\rho\left(t^{*}-\varepsilon\right)}\right) .
$$

Hence

$$
\log \left|a_{n}\right|>\frac{\lambda_{n}}{\rho}-\lambda_{n} \log \phi\left(\frac{\lambda_{n}}{\rho\left(t^{*}-\varepsilon\right)}\right)
$$

or

$$
\begin{aligned}
\log \left|a_{n}\right|^{-1 / \lambda_{n}} & <\log \left(\frac{\phi\left(\lambda_{n} / \rho\left(t^{*}-\varepsilon\right)\right)}{e^{1 / \rho}}\right) \\
& \simeq \log \phi\left(\lambda_{n} / e \rho\left(t^{*}-\varepsilon\right)\right)
\end{aligned}
$$

or for all large values of $n$,

$$
\left|a_{n}\right|^{-1 / \lambda_{n}}<\phi\left(\frac{\lambda_{n}}{e \rho\left(t^{*}-\varepsilon\right)}\right) \simeq \frac{\phi\left(\lambda_{n}\right)}{\left(e \rho\left(t^{*}-\varepsilon\right)\right)^{1 / \rho}} .
$$

On proceeding to limits, we have

$$
e \rho t^{*} \leq \liminf _{n \rightarrow \infty}\left(\frac{\phi\left(\lambda_{n}\right)}{\left|a_{n}\right|^{-1 / \lambda_{n}}}\right)^{\rho} .
$$

In case of $(p, q)=(2,2), r$ is chosen so that

$$
(\log r)^{\rho(r)-1}=\frac{\lambda_{n}}{\rho\left(t^{*}-\varepsilon\right)} \Longleftrightarrow \log r=\phi\left(\frac{\lambda_{n}}{\rho\left(t^{*}-\varepsilon\right)}\right) .
$$

Using this in $(2.13)$ when $(p, q)=(2,2)$, we get

$$
\begin{aligned}
\log \left|a_{n}\right| & >\left(t^{*}-\varepsilon\right) \log r^{\rho(r)}-\lambda_{n} \log r \\
& =\left(\frac{\lambda_{n}}{\rho}-\lambda_{n}\right) \log r=-\left(\frac{\rho-1}{\rho}\right) \lambda_{n} \log r .
\end{aligned}
$$

Thus,

$$
\begin{aligned}
\log \left|a_{n}\right|^{-1 / \lambda_{n}} & <\left(\frac{\rho-1}{\rho}\right) \phi\left(\frac{\lambda_{n}}{\rho\left(t^{*}-\varepsilon\right)}\right) \\
& \simeq\left(\frac{\rho-1}{\rho}\right) \frac{\phi\left(\lambda_{n}\right)}{\left(\rho\left(t^{*}-\varepsilon\right)\right)^{1 /(\rho-1)}} .
\end{aligned}
$$


Proceeding to limits as $n \rightarrow \infty$, we have

$$
\rho t^{*} \leq\left(\frac{\rho-1}{\rho}\right)^{\rho-1} \liminf _{n \rightarrow \infty}\left(\frac{\phi\left(\lambda_{n}\right)}{\log \left|a_{n}\right|^{-1 / \lambda_{n}}}\right)^{\rho-1} .
$$

The inequalities (2.14) and (2.15) combine to give (2.10).

For the case $p \geq 3$, let $\left|a_{n_{1}}\right| r^{\lambda_{n_{1}}}$ and $\left|a_{n_{2}}\right| r^{\lambda_{n_{2}}}$ be two consecutive maximum terms of $f(z)$. Then $n_{1} \leq n_{2}-1$ and by (2.12), $\left|a_{n_{1}}\right| r^{\lambda_{n_{1}}}$ is maximum term of $f(z)$ for $\psi\left(n_{1}-1\right) \leq r<\psi\left(n_{1}\right)$. Further, in view of Lemma 3, for these values of $r$ and for every $c>0$, we have

$$
\log ^{[p-2]} \nu(r)=\log ^{[p-2]} \lambda_{n_{1}}>\left(t^{*}-\varepsilon\right)\left(\log ^{[q-1]}\left(\psi\left(n_{1}\right)-c\right)\right)^{\rho(r)} .
$$

Thus, for $n_{1}<n \leq n_{2}, \psi\left(n_{1}\right)=\psi\left(n_{1}+1\right)=\cdots=\psi(n-1)$ and

$$
\log ^{[p-2]} \lambda_{n-1}>\left(t^{*}-\varepsilon\right)\left(\log ^{[q-1]}(\psi(n-1)-c)\right)^{\rho(r)} .
$$

As $\psi$ is increasing it can be proved that

$$
\log \left|a_{n}\right|^{-1 / \lambda_{n}}<(1+o(1)) \log \psi(n-1)
$$

Operating the function $\phi$ on (2.16), it follows that

$$
\frac{\phi\left(\log ^{[p-2]} \lambda_{n-1}\right)}{\log ^{[q-1]} \psi(n-1)}>\left(t^{*}-\varepsilon\right)^{1 / \rho} .
$$

The above inequality in conjunction with (2.17) gives (2.9).

Now, Propositions 1 and 2 lead to the following result.

Theorem 1. Let $f(z)$ be an entire function with index-pair $(p, q), p \geq q \geq 1$ and generalized lower $(p, q)$-type $t^{*}$ with respect to a proximate order $\rho(r)$ such that $\psi(n)=\left|a_{n} / a_{n+1}\right|^{1 /\left(\lambda_{n+1}-\lambda_{n}\right)}$ forms a nondecreasing function of $n$ for $n>n_{o}$. Then

$$
t^{*}=\liminf _{n \rightarrow \infty}\left[\frac{\phi\left(\log ^{[p-2]} \lambda_{n-1}\right)}{\log ^{[q-1]}\left|a_{n}\right|^{-1 / \lambda_{n}}}\right]^{\rho}, \quad p \geq 3 .
$$

The relation (2.18) holds for $p=2$ also, if $\lambda_{n-1} \simeq \lambda_{n}$ as $n \rightarrow \infty$, we have

$$
\frac{t^{*}}{M}=\liminf _{n \rightarrow \infty}\left[\frac{\phi\left(\lambda_{n-1}\right)}{\log ^{[A]}\left|a_{n}\right|^{-1 / \lambda_{n}}}\right]^{\rho-A},
$$


where $M$ has the same meaning as in Propositions 1 and 2.

Remark. Let us define $\rho(r)=\rho \forall r \in\left[\exp ^{[q-1]} 1, \infty\right)$ and $\phi(x)=x^{1 /(\rho-A)}$. Then, the following corollary is an answer to the queries raised in Theorem A.

Corollary. Let $f(z)$ be an entire function with index-pair $(p, q), p \geq q \geq 1$ and lower $(p, q)$-type $t$ such that $\psi(n)=\left|a_{n} / a_{n+1}\right|^{1 /\left(\lambda_{n+1}-\lambda_{n}\right)}$ forms a nondecreasing function of $n$ for $n>n_{o}$. Then

$$
\frac{t}{M}=\liminf _{n \rightarrow \infty} \frac{\log ^{[p-2]} \lambda_{n-1}}{\left(\log ^{[q-1]}\left|a_{n}\right|^{-1 / \lambda_{n}}\right)^{\rho}}, \quad p \geq 3,
$$

and this holds for $p=2$ also, if $\lambda_{n-1} \simeq \lambda_{n}$ as $n \rightarrow \infty$.

Finally, we prove the result for which the condition on $\psi(n)$ in Theorem 1 is not required.

THeorem 2. For an entire function $f(z)$ with index-pair $(p, q), p \geq q \geq 1$ and generalized $(p, q)$-type $t^{*}$ in reference to a proximate order $\rho(r)$, we have

$$
t^{*}=\max _{\left\{m_{k}\right\}}\left\{\liminf _{k \rightarrow \infty}\left[\frac{\phi\left(\log ^{[p-2]} \lambda_{m_{k-1}}\right)}{\log ^{[q-1]}\left|a_{m_{k}}\right|^{-1 / \lambda_{m_{k}}}}\right]^{\rho}\right\}, \quad \text { for } p \geq 3 .
$$

Further, if $\left\{\lambda_{n_{k}}\right\}$ be the sequence of principal indices such that $\lambda_{n_{k-1}} \simeq \lambda_{n_{k}}$ as $k \rightarrow \infty$, then for $p=2$,

$$
\frac{t^{*}}{M}=\max _{\left\{m_{k}\right\}}\left\{\liminf _{k \rightarrow \infty}\left[\frac{\phi\left(\lambda_{m_{k-1}}\right)}{\log ^{[A]}\left|a_{m_{k}}\right|^{-1 / \lambda_{m_{k}}}}\right]^{\rho-A}\right\}
$$

The maximum is taken over all increasing sequences of positive integers $\left\{m_{k}\right\}$.

Proof. Let $\left\{\lambda_{n_{k}}\right\}$ be the sequence of principal indices of an entire function $f(z)$. Consider the function

$$
g(z)=\sum_{k=0}^{\infty} a_{n_{k}} z^{\lambda_{n_{k}}} .
$$

Since the Taylor expansion of $g(z)$ contains coefficients and exponents as the subsequences of $\left\{a_{n}\right\}_{n=0}^{\infty}$ and $\left\{\lambda_{n}\right\}_{n=0}^{\infty}$, respectively, it follows that $g(z)$ is an entire function and moreover, as the exponents are the sequence of principal indices of $f(z)$ the function $g(z)$ has the same maximum term and rank as that of $f(z)$ for every value of $r$. Hence $g(z)$ is of index-pair $(p, q),(p, q)$-order $\rho$ and generalized lower $(p, q)$-type $t^{*}$ (in view of Lemma 2 ). It can be easily seen that 
$\psi\left(n_{k}\right)=\left|a_{n_{k}} / a_{n_{k+1}}\right|^{1 /\left(\lambda_{n_{k+1}}-\lambda_{n_{k}}\right)}$ forms a nondecreasing function of $k$. Hence from (2.18), we have, for $p \geq 3$,

$$
t^{*}=\liminf _{k \rightarrow \infty}\left[\frac{\phi\left(\log ^{[p-2]} \lambda_{n_{k-1}}\right)}{\log ^{[q-1]}\left|a_{n_{k}}\right|^{-1 / \lambda_{n_{k}}}}\right]^{\rho} .
$$

Since (2.1) holds for every increasing sequence $\left\{m_{k}\right\}$, it follows that

$$
t^{*} \geq \max _{\left\{m_{k}\right\}}\left\{\liminf _{k \rightarrow \infty}\left(\frac{\phi\left(\log ^{[p-2]} \lambda_{m_{k-1}}\right)}{\log ^{[q-1]}\left|a_{m_{k}}\right|^{-1 / \lambda_{m_{k}}}}\right)^{\rho}\right\} .
$$

Comparing these two results we get (2.20).

Similarly, for $p=2$, taking (2.19) and Proposition 1 into account, (2.21) follows.

\section{References}

[1] S.K. Bajpai, G.P. Kapoor and O.P. Juneja, On entire functions of fast growth, Trans. Amer. Math. Soc., 203 (1975), 275-297.

[2] O.P. Juneja, G.P. Kapoor and S.K. Bajpai, On the $(p, q)$-order and lower $(p, q)$-order of an entire function, J. Reine Angew. Math., 282 (1976), 53-67.

[3] O.P. Juneja, G.P. Kapoor and S.K. Bajpai, On the $(p, q)$-type and lower $(p, q)$-type of an entire function, J. Reine Angew. Math., 290 (1977), 180-190.

[4] H.S. Kasana and A. Sahai, The proximate order of entire Dirichlet series, Complex Variables: Theory and Application (New York-USA), 9-1 (1987), 49-62.

[ 5 ] H.S. Kasana, The existence theorem for proximate order of analytic functions, Ukrain. Mat. Zh. (Kiev), 40-1 (1988), 117-121.

[6] H.S. Kasana, The generalized type of entire functions with index-pair $(p, q)$, Comment. Math. Prac Mat., 29-2 (1990), 215-222.

[ 7 ] P. Lockhart and E.G. Straus, Relations between the maximum modulus and maximum term of an entire function, Pacific J. Math., 118-2 (1988), 479-483.

[ 8 ] S.M. Einstein-Matthews and H.S. Kasana, Proximate order and type of entire functions of several complex variables, Israel J. Math., 92 (1995), 273-284.

[ 9 ] D. Sato, On the rate of growth of entire functions of fast growth, Bull. Amer. Math. Soc., 69 (1963), 411-414.

Department of Mathematics \& Computer Applications Thapar Institute of Engineering and Technology Patiala-147 001, (Pb.), India 